\title{
Ecthyma gangrenosum aggravated by systemic antibiotics: A case report and literature review
}

\author{
Bibush Amatya', Rishabh Dev Tripathee', Rashmi Sharma', Leison Maharjan² \\ ${ }^{1}$ Resident, Dermatology, Institute of Medicine, Tribhuvan University Teaching Hospital, Kathmandu, Nepal, ${ }^{2}$ Resident, \\ Otorhinolaryngology, Institute of Medicine, Tribhuvan University Teaching Hospital, Kathmandu, Nepal
}

Corresponding author: Dr. Bibush Amatya, E-mail: doc_bibush@hotmail.com

\begin{abstract}
Ecthyma gangrenosum is a cutaneous manifestation of systemic infection caused predominantly by Pseudomonas aeruginosa. We report a case of ecthyma gangrenosum in a child caused by P. aeruginosa who had been previously unsuccessfully treated with systemic antibiotics. A four years old boy presented with the complaints of fever and ulcers on the trunk and extremities. He had been initiated on systemic antibiotics without sending or awaiting reports of blood and skin swab culture and sensitivity. The swab samples taken from the ulcers revealed growth of P. aeruginosa. He was then started on antipseudomonal intravenous antibiotics which eventually led to full recovery. Injudicious use of systemic antibiotics can lead to colonization and infection by opportunistic organisms such as P. aeruginosa. The use of antibiotics has to be based on reports of culture and sensitivity.
\end{abstract}

Key words: Ecthyma Gangrenosum; Vancomycin; Superinfection

\section{INTRODUCTION}

Ecthyma gangrenosum is a type of cutaneous lesion usually caused by Pseudomonas aeruginosa [1]. Although previously thought to occur primarily due to P. aeruginosa infection, newer studies [2] have revealed the condition to be caused by other bacteria $[3,4]$ and fungi $[5,6]$ too. The lesion starts as an erythematous papule, which slowly evolves to form a haemorrhagic blister, which then deroofs to reveal a characteristic ulcer with dark necrotic eschar and surrounding erythema [1]. We present a report of a four year old boy with ecthyma gangrenosum secondary to $P$. aeruginosa and Staphylococcus aureus infections, which resolved after a triple combination of intravenous antibiotics.

\section{CASE REPORT}

A four years old boy presented to the paediatric emergency with the complaints of fever and ulcers on trunk and extremities for 10 days. The patient was apparently well 10 days back, when he developed fever. On the same day as the onset of fever, he developed a small (pea sized) fluid filled lesion on the dorsum of his right hand. It was painful and associated with raised temperature. In the next three days, similar lesions developed on the trunk and bilateral upper and lower extremities. The fluid filled lesions ruptured in the next two to three days leaving a deep ulcer with dark underlying base and redness in the skin surrounding the ulcers.

He was taken to a subhealth post the next day and was given topical framycetin and oral flucloxacillin by a community health worker. However, there was no improvement in the appearance of the lesions and was further complicated by appearance of similar lesions on other body sites. After 5 days, he was taken to a pharmacy, where he was started on vancomycin intravenously and topical application of fusidic acid empirically. The therapy was continued for 3 days without any improvement after which the family members of the patient decided to visit our hospital.

His temperature at the time of admission was $101^{\circ} \mathrm{F}$, heart rate 138 beats per minute, respiratory rate 24 per

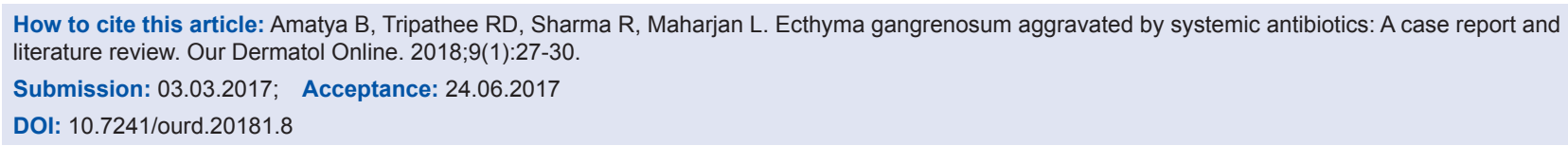


minute and blood pressure was 100/70 mm Hg with pallor noted on lower palpebral conjunctiva. His systemic examination did not reveal any abnormalities. His cutaneous examination revealed multiple circular small to large deep ulcers ranging from $1 \mathrm{~cm} \mathrm{X} 1 \mathrm{~cm}$ to $4 \mathrm{~cm} \mathrm{X} 4 \mathrm{~cm}$ on the trunk (Figure 1) and extremities (Figure 2) with black necrotic eschar, purulent exudates and erythema in surrounding skin.

Based on the history and clinical appearance of the lesions, a provisional diagnosis of ecthyma gangrenosum was made.

His baseline haematological and biochemistry investigations revealed the following findings:

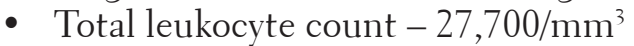

- Differential leukocyte count - Neutrophils 78\%, Lymphocytes 20\%, Monocytes 2\%

- ESR - $50 \mathrm{~mm}$ in $1^{\text {st }}$ hour.

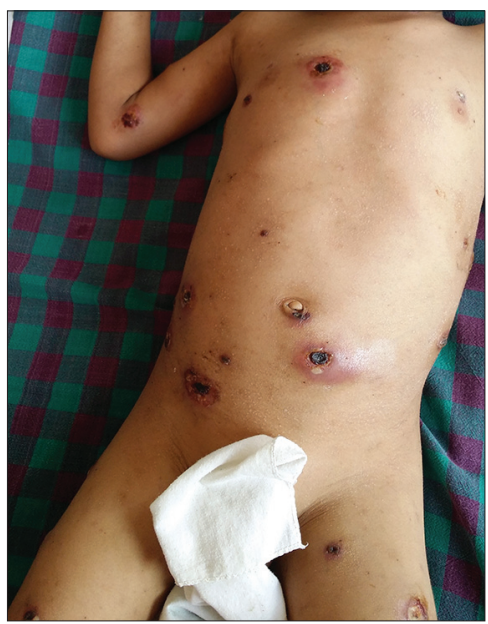

Fig. 1: Deep seated ulcers with black necrotic eschar and surrounding erythema on the trunk.

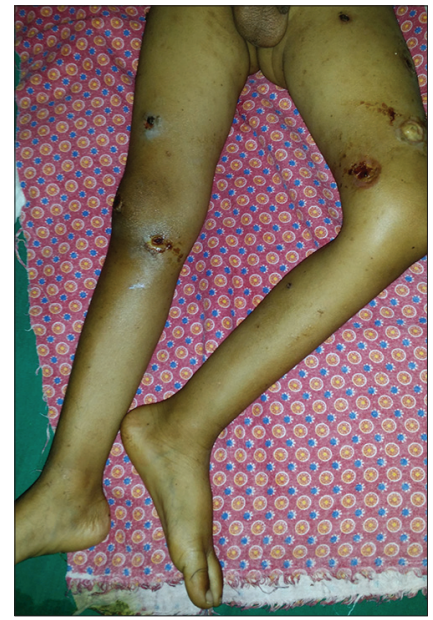

Fig. 2: Similar ulcers on the lower extremities.
- CRP - Positive.

- Biochemical parameters were within normal limits

- Blood culture and sensitivity and swab culture and sensitivity were sent.

The treatments initiated at the time were injection cloxacillin $250 \mathrm{mg}$ IV QID, syrup paracetamol $(125 \mathrm{mg} / 5 \mathrm{ml}) 6 \mathrm{ml}$ QID, daily dressing with potassium permanganate 1:10,000 diluted solution and application of mupirocin ointment twice daily.

Three days later, his condition had not improved. His blood culture did not reveal growth of any organism while the skin swab revealed isolation of Staphylococcus aureus sensitive to Tigecycline, Doxycycline, Clindamycin, Vancomycin and Chloramphenicol while resistant to Cloxacillin, Cefadroxil, Erythromycin, Ciprofloxacin, Levofloxacin and Amoxycillin.

In light of the swab culture and sensitivity report, cloxacillin was stopped and Injection Vancomycin $100 \mathrm{mg}$ IV QID was initiated, and haematology, biochemistry and swab culture and sensitivity were repeated.

Three days later, his condition improved marginally and the repeated culture revealed isolation of Pseudomonas aeruginosa sensitive to Amikacin, Tazobactam + Piperacillin, Polymyxin B but resistant to Ciprofloxacin, Levofloxacin, Gentamicin.

His diagnosis was revised as swab culture revealed the growth of Pseudomonas aeruginosa. He was subsequently put on injection Amikacin 150 mg IV OD and injection Tazobactam+Piperacillin $1 \mathrm{~g}$ IV TDS. The topical therapy was changed to a triple combination of Neomycin, Polymyxin B and Bacitracin.

Three days later, there was marked improvement in his symptoms with resolution of fever and associated improvement in the appearance of cutaneous lesions (Figure 3). The repeated swab culture revealed growth of Candida species other than Candida albicans.

The same treatment was continued for another 7 days during which time the lesions had started to heal with absence of exudates and black necrotic eschar. He was discharged on the $10^{\text {th }}$ day with all systemic medications stopped and was advised dressing of the healing wounds twice daily at home with normal saline followed by application of triple combination antibiotic (Neomycin/Polymyxin B/Bacitracin) ointment till full resolution. 


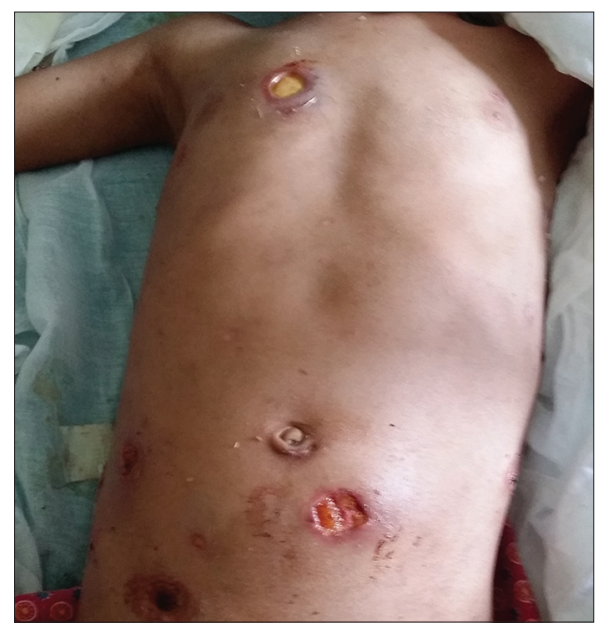

Fig. 3: Appearance of lesions while on treatment.

\section{DISCUSSION}

Ecthyma gangrenosum is one of the cutaneous manifestations of severe systemic Pseudomonas infection [1]. Previously, ecthyma gangrenosum was assumed to be caused solely by P. aeruginosa and that too only in the immunocompromised patients [2].

However, the paradigm has slowly changed in the last 40 years. Ecthyma gangrenosum like lesions have been known to be caused by other bacteria including Escherichia coli [3], Klebsiella pneumoniae [4] and Pseudomonas stutzeri [5]. The fungi known to cause these characteristic lesions include Candida [6], Fusarium [7], Mucor [8], Scytalidium [9] and Metarhizium [10].

The condition can occur with [11] or without bacteraemia [12] in both immunocompetent [13] and immunocompromised individuals [14]. The diagnosis is based on the appearance of characteristic deep ulcers with dark necrotic eschar on the base and erythema in the surrounding skin associated with a picture of leukocytosis or leukopenia in the total and differential blood count, isolation of the causative organisms in blood or wound culture and improvement of the condition after institution of systemic antibiotics or antifungals based on the culture and sensitivity reports.

The difficulty in our case lay in the isolation of two different organisms, $S$. aureus and $P$. aeruginosa from two distinct sites. Our patient had already been initiated on vancomycin at another centre but had not improved while on vancomycin. As the first swab culture revealed growth of $S$. aureus sensitive to vancomycin, our patient was re-started on vancomycin.
However, the degree of clinical improvement while on vancomycin was not appreciable.

The isolation of $P$. aeruginosa from the second swab site three days after the detection of $S$. aureus can be explained in two different ways. The first is co-infection by Pseudomonas aeruginosa with $S$. aureus, with the characteristic ulcers being a feature of Pseudomonas infection. Another theory is the secondary colonization of the ulcer bed by Pseudomonas after removal of the Gram positive organisms by first systemic cloxacillin and then by systemic vancomycin. This is especially relevant in Nepal where systemic antibiotics are available over the counter resulting in their indiscriminate use.

The combination of two systemic agents is recommended for pseudomonal infection, because of higher rate of morbidity and mortality associated with this infection. As the combination of tazobactam-piperallicin and amikacin shows the greater synergy [15], the two agents were systemically administered in our patient. The reports of the wound swab culture and sensitivity also showed the organism to be susceptible to both the agents. Similarly, the use of topical antibiotic combination of neomycin/bacitracin/polymyxin B was also guided by the results of wound swab culture and sensitivity.

\section{CONCLUSION}

Ecthyma gangrenosum is usually caused by infection with Pseudomonas aeruginosa. However, similar lesions were caused in our patient by co-infection with Staphylococcus aureus and P. aeruginosa. Pseudomonal colonization and infection can occur after systemic treatment for Gram positive organisms. The treatment approach has to be based on the results of blood and wound swab culture and sensitivity. A combination of systemic vancomycin for $S$. aureus infection, amikacin and piperacillin-tazobactam for P. aeruginosa infection was effective in treating the deep ulcers of ecthyma gangrenosum.

Injudicious use of systemic antibiotics can lead to colonization and infection by other organisms. This is especially relevant in Nepal where administration of systemic antibiotics do not require prescription by health care providers. Stricter laws preventing over the counter sale of systemic antibiotics may help curtail these problems. 


\section{ACKNOWLEDGEMENTS}

The authors would like to thank the guardians of the subject for granting their approval to write and publish this case report. The authors are also grateful to the faculty members and residents of the Department of Dermatology and Venereology at the Institute of Medicine, Tribhuvan University Teaching Hospital, Maharajgunj, Kathmandu, Nepal for their encouragement in preparing this case report.

\section{REFERENCES}

1. Boisseau AM, Sarlangue J, Perel Y, Hehunstre JP, Taieb A, Maleville J. Perineal ecthyma gangrenosum in infancy and early childhood: septicemic and nonsepticemic forms. J Am Acad Dermatol. 1992;27:415-8.

2. Vaiman M, Lazarovitch T, Heller L, Lotan G. Ecthyma gangrenosum and ecthyma like lesions: review article. Eur J Clin Microbiol Infect Dis. 2014;34:633-9.

3. Edelstein H, Cutting HO. Escherichia coli as cause of ecthyma gangrenosum. Postgrad Med. 1986;79:44-5.

4. Rodot S, Lacour JP, van Elslande L, Castanet J, Desruelles F, Ortonne JP. Ecthyma gangrenosum caused by Klebsiella pneumoniae. Int J Dermatol. 1995;34:216-7.

5. Puzenat E, Chirouze C, Khayat N, Aubin F, Estavoyer JM, Humbert P, Hoen B. Ecthyma gangrenosum caused by Pseudomonas stutzeri with bacteraemia and systemic vascularitis. Rev Med Interne. 2004;25:315-8.

6. Agarwal S, Sharma M, Mehndirata V. Solitary ecthyma gangrenosum (EG)-like lesion consequent to Candida albicans in a neonate. Indian J Pediatr. 2007;74:582-4.

7. Uludokumacı S, Balkan II, Mete B, Ozaras R, Saltoğlu N, Soysal T. Ecthyma gangrenosum-like lesions in a febrile neutropenic patient with simultaneous Pseudomonas sepsis and disseminated fusariosis. Turk J Haematol. 2013;30:321-4.

8. Kramer BS, Hernandez AD, Reddick RL, Levine AS. Cutaneous infarction: Manifestation of disseminated mucormycosis. Arch Dermatol. 1977;113:1075-6.

9. Benne CA, Neeleman C, Bruin M, de Hoog GS, Fleer A. Disseminating infection with Scytalidium dimidiatum in a granulocytopenic child. Eur J Clin Microbiol Infect Dis. 1993;12:118-21.

10. Burgner D, Eagles G, Burgess M, Procopis P, Rogers M, Muir D, et al. Disseminated invasive infection due to Metarrhizium anisopliae in an immunocompromised child. J Clin Microbiol. 1998;36:1146-50.

11. Duman M, Ozdemir D, Yiş U, Köroğlu TF, Oren O, Berktaş S. Multiple erythematous nodules and ecthyma gangrenosum as a manifestation of Pseudomonas aeruginosa sepsis in a previously healthy infant. Pediatr Dermatol. 2006;23:243-6.

12. Gençer S, Ozer S, Ege Gül A, Doğan M, Ak O. Ecthyma gangrenosum without bacteremia in a previously healthy man: A case report. J Med Case Rep. 2008;2:14.

13. Fabrizi G, Pagliarello C. Multiple ecthyma gangrenosum with a favorable course in a nonimmunocompromised child with moyamoya disease. Eur J Dermatol. 2007;17:253-4.

14. Techatawepisarn T, Chiewchanvit S, Salee P, Mahanupab P, Baosoung V, Praparattanapan J. Ecthyma gangrenosum-like lesions associated with disseminated nontuberculous mycobacterial infection in an HIV-infected patient. Southeast Asian J Trop Med Public Health. 2013;44:649-54.

15. Burgess DS, Hastings RW. Activity of piperacillin/tazobactam in combination with amikacin, ciprofloxacin, and trovafloxacin against Pseudomonas aeruginosa by time-kill. Diagn Microbiol Infect Dis. 2000;38:37-41.

Copyright by Bibush Amatya, et al. This is an open-access article distributed under the terms of the Creative Commons Attribution License, which permits unrestricted use, distribution, and reproduction in any medium, provided the original author and source are credited.

Source of Support: Nil, Conflict of Interest: None declared. 